\title{
Drug-Induced Subacute Cutaneous Lupus Erythematosus Associated with Proton Pump Inhibitors
}

\author{
Nitish Aggarwal ${ }^{1}$
}

Published online: 29 March 2016

(c) The Author(s) 2016. This article is published with open access at Springerlink.com

\begin{abstract}
Subacute cutaneous lupus erythematosus (SCLE) is an autoimmune disease that may be induced by proton pump inhibitors (PPIs) in at-risk populations. The US FDA does not recognize SCLE as an adverse event associated with PPIs. We queried the FDA Adverse Event Reporting System database, which contains adverse event case reports submitted by the public as well as by industry, and analyzed the data to quantify passive pharmacovigilance signals for SCLE associated with PPIs. A disproportionality analysis of the signals yielded a significant association between SCLE and PPIs. Discontinuation of PPI resulted in remission, with PPI re-challenge causing SCLE to reoccur. A follow-up analysis also yielded a significant association between SCLE and $\mathrm{H} 2$ receptor antagonists. We conducted a brief literature survey of published case reports and studies to discern the validity of PPI-induced SCLE signals. Healthcare prescribers and patients should be made aware that SCLE can be induced by PPIs. In such cases, PPIs should be discontinued and alternative clinical treatment sought. Regulatory bodies such as the FDA should incorporate the adverse reaction in PPI prescription labels.
\end{abstract}

Nitish Aggarwal

nitish.aggarwal@osumc.edu

1 The Ohio State University Wexner Medical Center, $410 \mathrm{~W}$ 10th Ave, Columbus, OH 43210, USA

\section{Key Points}

Pharmacovigilance analysis of the US FDA Adverse Event Reporting System (FAERS) database provided a signal of association between subacute cutaneous lupus erythematosus and proton pump inhibitors.

Statistical analysis and published case reports confirmed the association.

Proton pump inhibitors should be discontinued if subacute cutaneous lupus erythematosus symptoms occur.

\section{Introduction}

The rate of autoimmune disorders is increasing exponentially in the Western world. In the USA, the prevalence of autoimmune disease has risen from $3 \%$ in the 1960s to $9 \%$ in 2009 [1]. One of the autoimmune diseases is lupus erythematosus, in which a hyperactive immune system attacks its own tissue cells. Subacute cutaneous lupus erythematosus (SCLE) is a distinct subset of cutaneous lupus erythematosus and presents clinically with nonscarring, erythematous, annular polycyclic or papulosquamous cutaneous eruptions in sun-exposed areas [2]. While SCLE can be idiopathic or drug induced, they are generally immunologically, histopathologically, and clinically indistinguishable. Thiazides, terbinafine, calcium channel blockers, angiotensin-converting enzyme inhibitors (ACEIs), tumor necrosis factor (TNF)- $\alpha$ inhibitors, and chemotherapeutic agents have all been implicated as suspected or probable causes of drug-induced SCLE. Proton 
pump inhibitors (PPIs) have also been associated with and may induce SCLE.

The US FDA does not recognize SCLE as a PPI-associated adverse event, and FDA-approved prescribing information for PPIs does not include SCLE as an associated adverse event. In this article, we analyze passive pharmacovigilance signals for PPI-associated SCLE and support our findings with published case reports and casecontrol studies. PPIs as a class work by inhibiting gastric acid secretion in the gastric lumen. They inhibit the $\mathrm{K}+/$ $\mathrm{H}+$ ATPase pump in the lining of gastric parietal cells [3]. This causes a reduction in acid secretion because hydrogen ions are unable to be transported to the gastric surface. PPIs are used to treat conditions such as dyspepsia and gastroesophageal reflux disease (GERD). This group of inhibitors comprises some of the World Health Organization (WHO) "World's essential medications" such as omeprazole, pantoprazole, and lansoprazole.

\section{Methods}

\subsection{Data Collection}

The FDA Adverse Event Reporting System (FAERS) database collects spontaneous reports of adverse events and medication errors involving human drugs and therapeutic biological products. The information is publically available as computerized quarterly data reports on the FAERS website [4]. Adverse event and medication error reports are submitted to the FDA by drug manufacturers, healthcare professionals (e.g., physicians, pharmacists, and nurses), and consumers (e.g., patients, family members, and lawyers). The original Adverse Event Reporting System (AERS) was designed in 1969 to support the FDA's postmarketing safety surveillance program for drug and therapeutic biologic products. It was replaced by FAERS on 10 September 2012, and the database now contains over 9 million reports of adverse events from 1969 to the present day. Since the last major revision, in 1997, reporting has markedly increased. The quarterly data files, available in ASCII or SGML formats, include demographic and administrative information; drug, reaction, and patient outcome information from the reports; and information on the source of the reports [4].

The adverse events data for the present analysis were obtained from the FAERS website for the period 1 July 2013 to 30 June 2015. The data tables "Demographics," "Drugs," "Indications," "Outcomes, Reactions," "Report Source," and "Therapy" were downloaded and imported into SQL Server (Microsoft SQL Server 2015); we then combined the files using primary key and foreign keys. The resulting tables were cleaned and duplicates removed. The data were queried using SQL queries.

\subsection{Adverse Event and Exposure Drug}

We investigated the adverse event "subacute cutaneous lupus erythematosus" and the drug class PPIs as being associated with SCLE. The PPI class was defined as comprising any of the following drugs: esomeprazole, dexlansoprazole, omeprazole, lansoprazole, pantoprazole, or rabeprazole, singularly or in combination.

\subsection{Analysis}

Disproportionality analysis can be used to identify statistical associations between products and events in their respective safety report databases. Such analysis compares the observed count for a product-event combination with an "expected" count. Unexpectedly high reporting associations "signal" that there may be a causal association between the particular adverse event and the product. This analysis can also identify increased reporting rates for low frequency events.

We used disproportionality analysis to quantify pharmacovigilance signals in FAERS to provide a picture of the drug-related risk. The association between the adverse event and the drugs of interest was evaluated using the Chi squared $\left(\chi^{2}\right)$ value with Yates correction, with values $>4$ considered statistically significant. We also calculated measurements of disproportionality (observed-expected ratios): relative reporting ratio (RRR), proportional reporting ratio (PRR), and reporting odds ratio (ROR), and their $95 \%$ confidence intervals. Generally, the higher the ratios, the more likely the association between $\operatorname{drug}(\mathrm{s})$ and adverse event(s). According to the criteria of Evans et al. [5], if (number of events $\geq 3, \chi^{2} \geq 4$, and PRR $\geq 2$ ), the combination of $\operatorname{drug}(\mathrm{s})$ and adverse event(s) is considered "likely" to be an adverse reaction.

\section{Results}

\subsection{Analysis Results}

A total of 220 cases listing SCLE as an adverse event were reported during the 2-year period (third quarter of 2013 to second quarter of 2015) with the use of any drug. Of these, 190 were reported by healthcare professionals and four by consumers; none were reported by lawyers. The source for the remaining 26 was missing from the database. Table 1 summarizes the major drug classes reported to have been associated with SCLE during the period, and Table 2 
presents the demographics of the patients for whom the adverse events were reported.

The query in the SQL data tables identified that more than half (120) of the 220 SCLE adverse events were associated with PPIs. Of the 120 cases associated with PPIs, 95 were reported by healthcare professions and two by consumers; none were reported by lawyers. The source for the remaining 24 was missing from the database. Of the 120 adverse events, $95 \%$ were coded as serious, with death reported as an outcome in $4.2 \%$. PPIs were identified as the "primary suspect" drug in all 120 adverse events. Table 3 presents a $2 \times 2$ table of the queried results; $\chi^{2}$ with Yates correction was 1874.74.

Table 1 The top ten drug classes associated with subacute cutaneous lupus erythematosus reported in the US FDA Adverse Event Reporting System database between 1 July 2013 and 30 June 2015, stratified by drug class

\begin{tabular}{lll}
\hline S. no. & Drug class & Reported SCLE cases $(n)$ \\
\hline 1 & PPI & 120 \\
2 & Statins & 34 \\
3 & Corticosteroids & 27 \\
4 & Thiazide diuretics & 25 \\
5 & ACEI/ARB & 22 \\
6 & Thyroid medications & 20 \\
7 & Aspirin & 16 \\
8 & Beta blocker & 15 \\
9 & Benzodiazepine & 15 \\
10 & Stool softener & 13 \\
\hline
\end{tabular}

$A C E I$ angiotensin-converting enzyme inhibitor, $A R B$ angiotensin receptor blocker, PPI proton pump inhibitor, SCLE subacute cutaneous lupus erythematosus
Table 4 presents the disproportionality ratios. A comparison of the results of the passive pharmacovigilance analysis using FAERS data $\left(n=120 ; \chi^{2}=1874.74\right.$, PRR $=36.64$ [95 \% CI 28.10-47.77]) and Evans et al. [5] criteria $\left(n \geq 3, \chi^{2} \geq 4\right.$, PRR $\left.\geq 2\right)$ indicates that SCLE is statistically significantly associated with PPIs.

Of the 120 PPI-associated SCLE cases, 68 reports indicate the drug was withdrawn after the event; no report indicated a drug reduction, and only one report indicated that drug dose remained unchanged. The other 51 cases reported no action or "unknown." Of the 120 PPI-induced SCLE cases, only nine were reported to have been followed longitudinally. All nine were reported to have reoccurred on re-administration of the drug.

We subsequently stratified the data by sex and obtained the corresponding disproportionality results. Table 3 presents a $2 \times 2$ table of the queried stratified results, and Table 4 presents the corresponding disproportionality ratios. The stratified results indicate that SCLE is statistically significantly associated with PPIs as an adverse event in women $\left(n=97 ; \chi^{2}=1591.27\right.$; PRR $=40.59[95 \% \mathrm{CI}$ 29.97-54.99]). A significant association is also seen in men, but to a lesser extent $\left(n=9 ; \quad \chi^{2}=61.72\right.$; $\mathrm{PRR}=13.85$ [95\% CI 6.18-31.07]).

When SCLE occurs and the PPI is discontinued but the patient still requires acid-suppression medication, one option is to prescribe $\mathrm{H} 2$ receptor antagonists such as cimetidine, famotidine, nizatidine, or ranitidine. We could not identify via the FAERS data whether an $\mathrm{H} 2$ receptor antagonist was started after a PPI was discontinued, and the ensuing outcome of that switch on SCLE. However, we did analyze FAERS data during the same time period to check for any independent association between SCLE and H2

Table 2 Demographics of patients with subacute cutaneous lupus erythematosus reported in the US FDA Adverse Event Reporting System database between 1 July 2013 and 30 June 2015

\begin{tabular}{lccc}
\hline Characteristic & Total $(n=220)$ & Associated with PPI $(n=120)$ & Associated with other drugs $(n=100)$ \\
\hline Sex ${ }^{\mathrm{a}}$ & & & $17(17.0)$ \\
Male & $26(11.8)$ & $9(7.5)$ & $73(73.0)$ \\
Female & $170(77.3)$ & $97(80.8)$ & $10(10.0)$ \\
Missing & $24(10.9)$ & $14(11.7)$ & $0(0)$ \\
Age (years) & & & $25(25.0)$ \\
$<25$ & $10(4.5)$ & $10(8.3)$ & $27(27.0)$ \\
$\geq 25$ and $<50$ & $50(22.7)$ & $25(20.8)$ & $37(37.0)$ \\
$\geq 50$ and $<70$ & $71(32.3)$ & $44(36.7)$ & $11(11.0)$ \\
$\geq 70$ and $<86$ & $56(25.5)$ & $19(15.8)$ & $2(2.0)$ \\
Missing & $33(15.0)$ & $22(18.3)$ & $5(4.2)$ \\
Mortality & $7(3.2)$ & & \\
\hline
\end{tabular}

Data are presented as $n(\%)$

$P P I$ proton pump inhibitor

a Fisher's exact test (PPI and 'other drugs' vs. 'male' and 'female': $p=0.0269$ [one tailed]) 
receptor antagonists (Tables 5, 6). The results indicate that SCLE is also significantly associated with $\mathrm{H} 2$ receptor antagonists, but to a much smaller extent $(n=5$;

Table $32 \times 2$ Contingency tables for reported proton pump inhibitor-associated subacute cutaneous lupus erythematosus

\begin{tabular}{|c|c|c|c|}
\hline Sex & PPIs & All other drugs & Total \\
\hline \multicolumn{4}{|l|}{ All } \\
\hline SCLE & 120 & 100 & 220 \\
\hline All other AEs & 56,604 & $1,732,098$ & $1,788,702$ \\
\hline Total AEs & 56,724 & $1,732,198$ & $1,788,922$ \\
\hline \multicolumn{4}{|c|}{$\chi^{2}$ with Yates correction: 1874.74} \\
\hline \multicolumn{4}{|l|}{ Female } \\
\hline SCLE & 97 & 73 & 170 \\
\hline All other AEs & 31,546 & 966,585 & 998,131 \\
\hline Total AEs & 31,643 & 966,658 & 998,301 \\
\hline \multicolumn{4}{|c|}{$\chi^{2}$ with Yates correction: 1591.27} \\
\hline \multicolumn{4}{|l|}{ Male } \\
\hline SCLE & 9 & 17 & 26 \\
\hline All other AEs & 22,810 & 597,071 & 619,881 \\
\hline Total AEs & 22,819 & 597,088 & 619,907 \\
\hline \multicolumn{4}{|c|}{$\chi^{2}$ with Yates correction: 61.72} \\
\hline
\end{tabular}

$A E$ adverse event, $P P I$ proton pump inhibitor, SCLE subacute cutaneous lupus erythematosus

Table 4 Disproportionality analysis results for reported proton pump inhibitor-associated subacute cutaneous lupus erythematosus

\begin{tabular}{llc}
\hline Sex & Measure & Ratio $(95 \%$ CI $)$ \\
\hline All & Proportional reporting ratio & $36.64(28.10-47.77)$ \\
& Relative odds ratio & $36.72(28.16-47.89)$ \\
& Relative reporting ratio & $17.20(13.77-21.48)$ \\
Female & Proportional reporting ratio & $40.59(29.97-54.99)$ \\
& Relative odds ratio & $40.71(30.05-55.17)$ \\
& Relative reporting ratio & $18.00(14.03-23.09)$ \\
Male & Proportional reporting ratio & $13.85(6.18-31.07)$ \\
& Relative odds ratio & $13.86(6.18-31.09)$ \\
& Relative reporting ratio & $9.40(4.41-20.07)$ \\
\hline
\end{tabular}

CI confidence interval

Table $52 \times 2$ Contingency table for reported H2 receptor antagonist-associated subacute cutaneous lupus erythematosus

\begin{tabular}{lccr}
\hline & $\begin{array}{l}\text { H2 receptor } \\
\text { antagonists }\end{array}$ & All other drugs & \multicolumn{1}{l}{ Total } \\
\hline SCLE & 5 & 215 & 220 \\
All other AEs & 11,057 & $1,777,645$ & $1,788,702$ \\
Total AEs & 11,062 & $1,777,860$ & $1,788,922$ \\
$\chi^{2}$ with Yates correction: 7.29 & & \\
\hline
\end{tabular}

$A E$ adverse event, $S C L E$ subacute cutaneous lupus erythematosus
Table 6 Disproportionality analysis results for reported $\mathrm{H} 2$ receptor antagonist-associated subacute cutaneous lupus erythematosus

\begin{tabular}{ll}
\hline Measure & Ratio (95 \% CI) \\
\hline Proportional reporting ratio & $3.74(1.54-9.07)$ \\
Relative odds ratio & $3.74(1.54-9.08)$ \\
Relative reporting ratio & $3.68(1.52-8.92)$
\end{tabular}

$\mathrm{H} 2$ receptor antagonist-associated SCLE cases $=7 / 220$

CI confidence interval

$\chi^{2}=7.29 ; \mathrm{PRR}=3.74[95 \%$ CI 1.54-9.07]) than with PPIs.

\subsection{Supporting Literature}

We conducted a brief review of the literature relating to PPI-associated SCLE. We searched PubMed for published studies (2000-2015) [6] to discern the validity of passive pharmacovigilance. The literature studies reviewed are broadly classified as clinical case reports, retrospective studies, and case-control studies.

\subsubsection{Clinical Case Reports}

We identified 22 clinical case reports from 21 patients (Table 7). The mean patient age was 58.33 years (range $30-85$ ), and 20 of the 21 patients were female. One patient was pregnant when she experienced the adverse reaction. The presence of antinuclear antibodies, including anti-Ro/ SSA antibodies and anti-La/SSB antibodies, in blood serum can be used to diagnose autoimmune disorders. Antinuclear antibodies, positive anti-Ro/SSA antibodies, and positive anti-La/SSB antibodies were found in 90, 90, and $36 \%$ of tested patients at the time of the rash, respectively. The incubation period was in the range of 3 days to 5 months (mean 5 weeks), and the resolution period was in the range of a few days to 4 months (mean 4.33 weeks) after the discontinuation of the PPI. SCLE recurred in two patients with PPI re-challenge and resolved after drug cessation. Two patients in whom the PPI was not stopped had active SCLE up to their deaths (death reported as due to other causes).

\subsubsection{Chart Review}

Sandholdt et al. [7] reported a retrospective medical chart review of patients with SCLE carried out at Odene University Hospital, Denmark. They identified 727 medical records with an International Statistical Classification of Diseases and Related Health Problems, tenth revision (ICD-10) diagnosis code of lupus erythematosus, 429 of which had confirmed cutaneous lupus erythematosus. In 


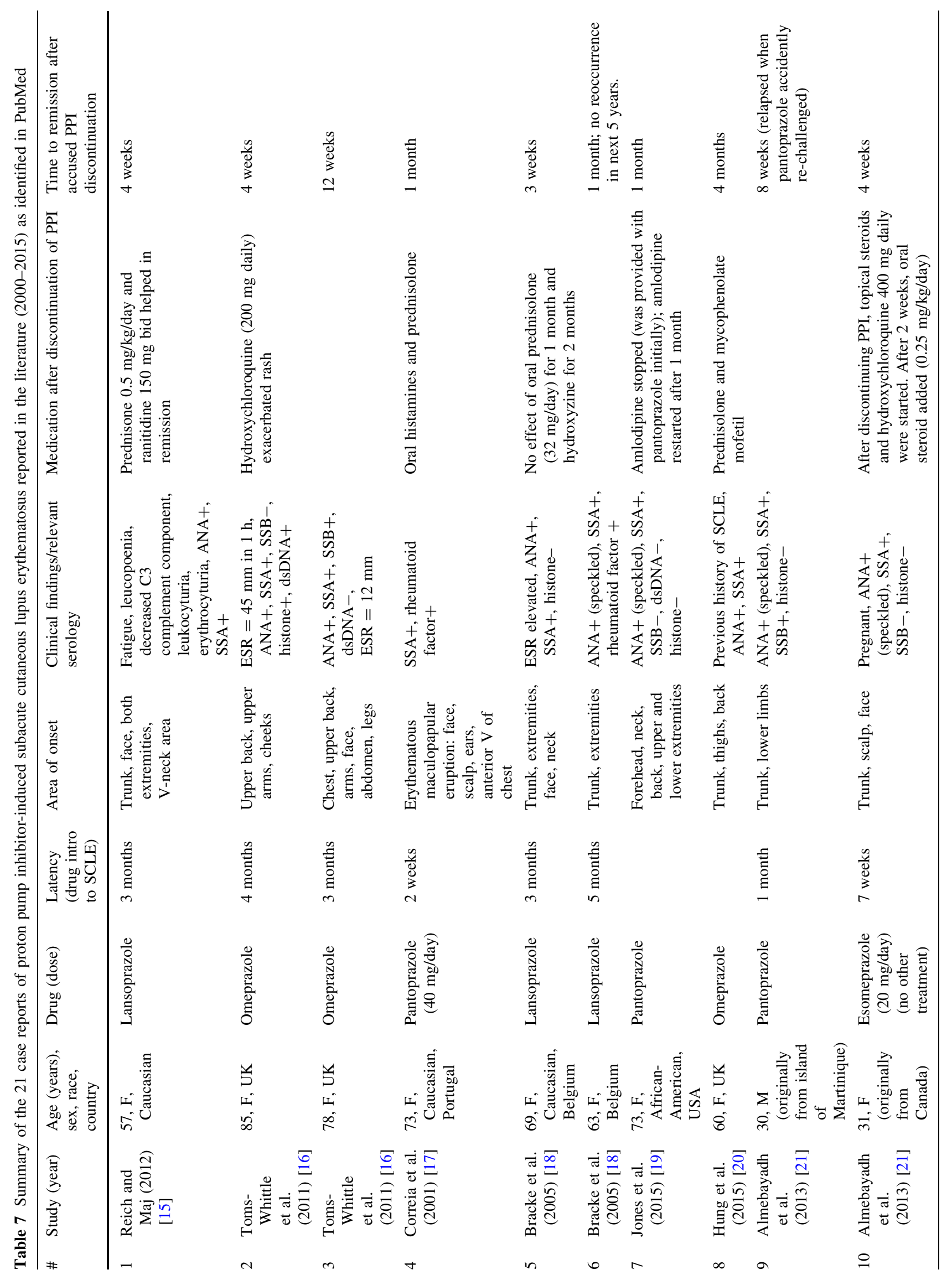




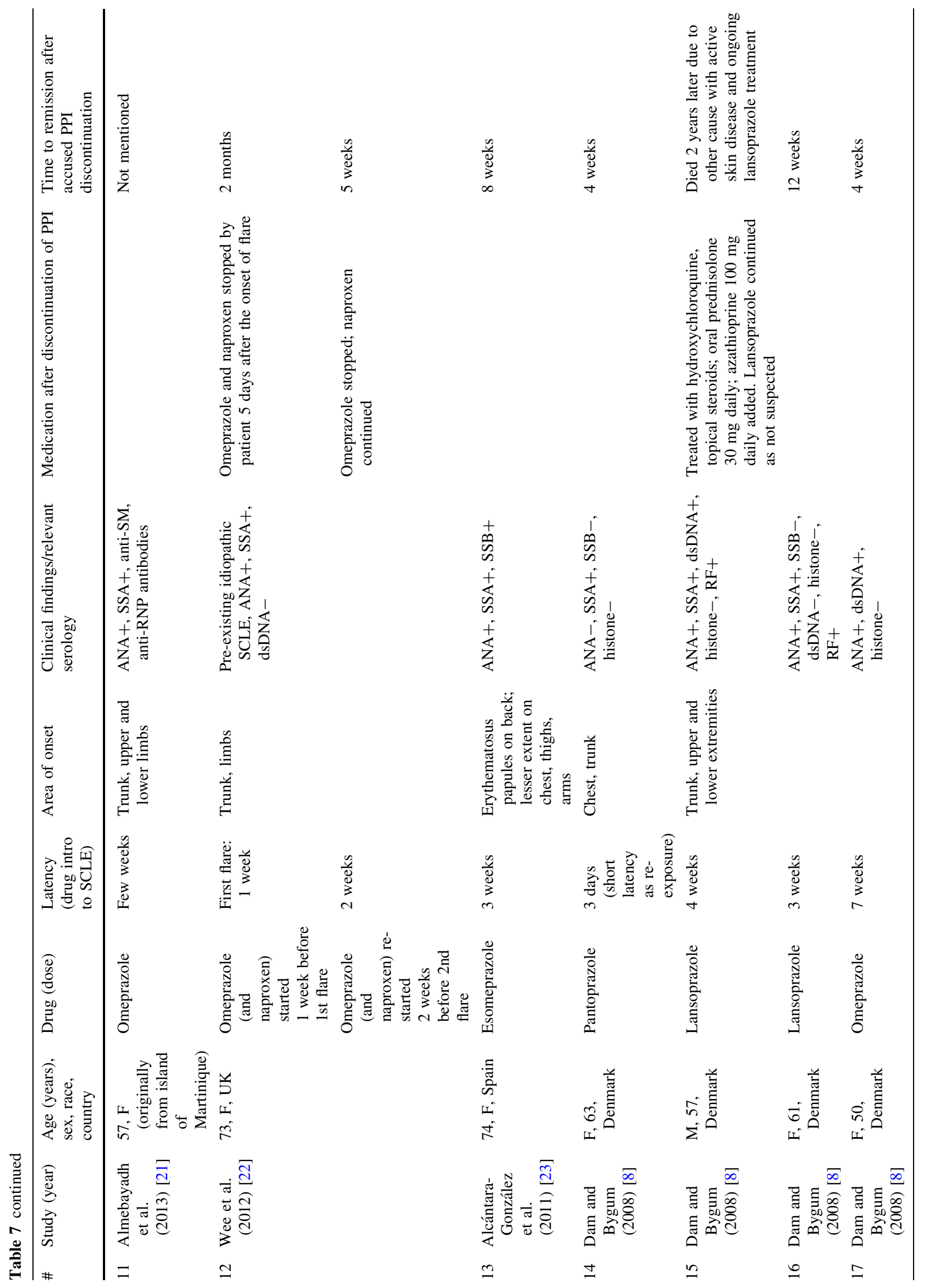




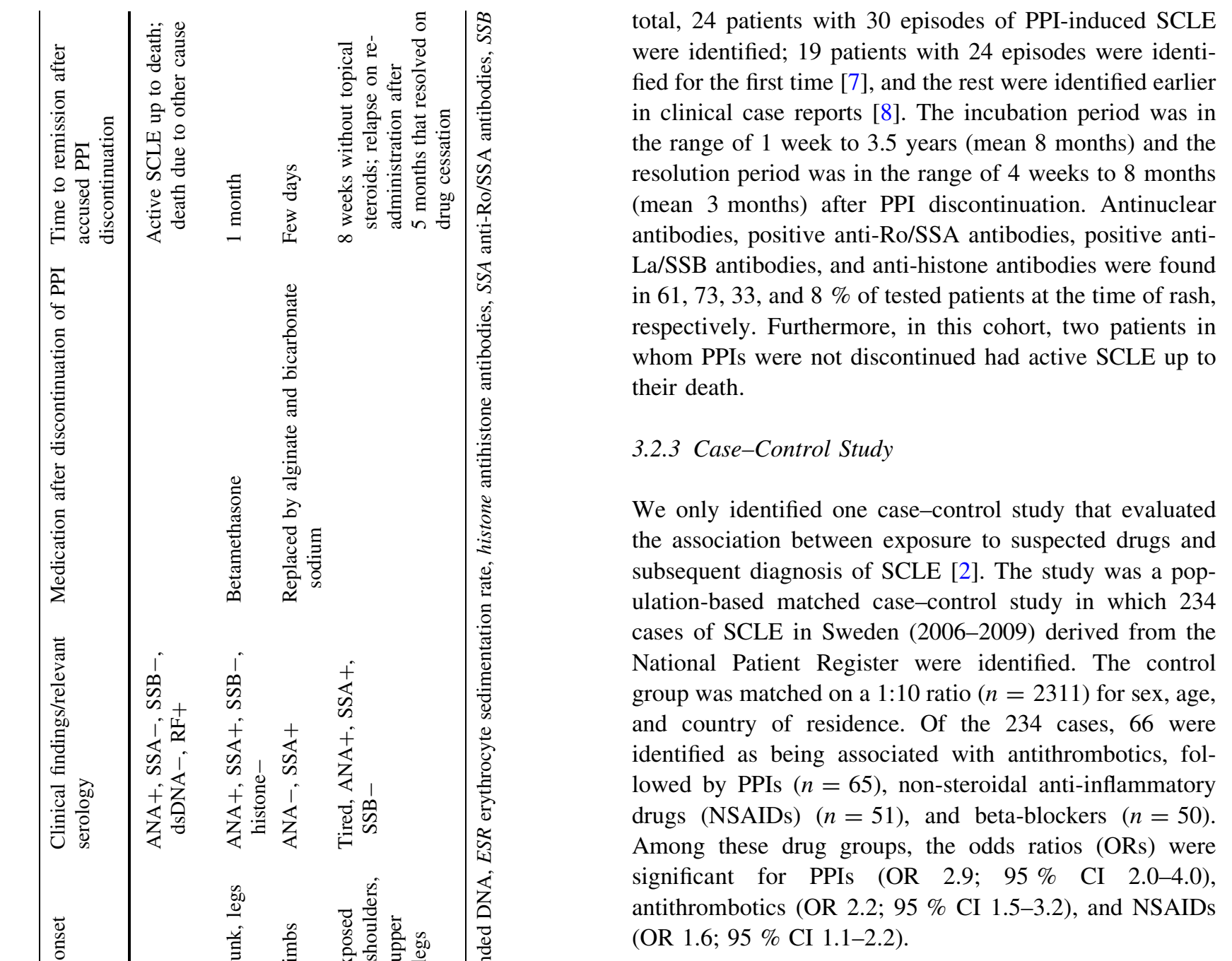

\section{Discussion}

\subsection{Limitations of Analysis}

FAERS provides a passive pharmacovigilance risk signal and indicates associations. However, there is no certainty that the reported event (adverse event) was actually induced or caused by the drug product. Although the disproportionality analysis quantifies the signal to provide a picture of the drug-related risk, it in itself is generally insufficient to prove a causal relationship. The analysis, coupled with multiple sources of data such as clinical case reports and case-control studies, can be helpful in inferring the causal relationship and recommending and modifying clinical practice guidelines. Regulatory actions in response to emerging drug safety concerns often depend on an accurate assessment of risks using multiple data sources as well as consideration of overall benefits and risks of the agent [9]. Therefore, this study should be placed in context with other such sources. 
Further, FDA does not receive reports for every adverse event or medication error that occurs with a product. Identical or similar reports may be submitted by a healthcare professional and the patient or lawyer. Many factors can influence whether or not an event will be reported, such as the time a product has been marketed and publicity about an event. Therefore, FAERS data cannot be used to calculate the incidence of an adverse event or medication error within a population [4]. Well designed, randomized, prospective, large-scale, and long-term clinical trials are the best way to assess the relationship between an adverse event and a drug. On the other hand, while post-marketing vigilance such as FAERS has a limited ability to establish causal relationships, it does have advantages in terms of being able to identify signals in real-world situations with multi-drug regimens, reactivations of adverse events after cessation of therapy, and global differences in the occurrence of adverse events.

\subsection{General Discussion}

Over the past decade, an increasing number of drugs in different classes have been implicated as triggers for druginduced SCLE. Antihypertensives (including calcium channel blockers, diuretics, beta-blockers, and ACEIs), antifungals, chemotherapeutics, antihistamines, immunomodulators, antiepileptics, statins, NSAIDs, hormone-altering drugs, and ultraviolet therapy have all been reported as inducing SCLE. One possibility is that many drugs trigger SCLE by inducing a photosensitive state. Such a state might then nonspecifically induce SCLE skin lesions via an isomorphic response (Köbner response) in an individual who is immunogenetically predisposed to developing SCLE [10].

In our analysis, 120 of the 220 SCLE cases reported in FAERS during the 2-year study period were associated with PPIs. The passive pharmacovigilance data indicated that PPIs were statistically significantly associated with lupus. However, it is possible that this is not a side effect of PPI but that a downstream mechanism is causing these symptoms. To explore this further, we compared PPIs and $\mathrm{H} 2$ receptor antagonists, an alternative to PPIs. PPIs work by inhibiting the $\mathrm{K}+\mathrm{H}+$ ATPase pump in the lining of the gastric parietal cells. This reduces acid secretion because the hydrogen ions are unable to be transported to the gastric surface. $\mathrm{H} 2$ receptor antagonists work in a similar manner and have the same end effect: inhibition of the proton pump. However, $\mathrm{H} 2$ receptor antagonist drugs initially block the histamine channel. This channel downregulates the proton pump via the vagus nerve. Since $\mathrm{H} 2$ receptors also statistically significantly cause this phenomenon, it is likely that the proton pump induces SCLE instead of SCLE being a direct side effect of PPIs.
The statistically significant findings from our analysis of FAERS signals indicate the signal generated by FAERS is not false positive and that the SCLE is induced by PPIs. This cause-effect relationship is reinforced by the recurrence of SCLE upon re-challenge with the PPI in all nine longitudinally followed FAERS cases. Similarly, in the published clinical case reports where cessation of PPI treatment resulted in remission, a re-challenge again resulted in SCLE. PPI re-challenge caused SCLE to reemerge with a shorter incubation period, reinforcing the relationship and indicating that an earlier episode of SCLE predisposes the patient to subsequent SCLE flare-ups.

At the Pharmacovigilance Risk Assessment Committee meeting in July 2015, the European Medicines Agency (EMA) issued a warning that SCLE is likely to be a class effect for PPIs [11]. The warning further states that SCLE after previous treatment with a PPI may increase the risk of SCLE with other PPIs. In September 2015, the UK Medicines and Healthcare products Regulatory Agency (MHRA) [12] stated that PPIs are associated with very infrequent cases of SCLE. These reports by the EMA and MHRA were based on the global safety databases of Takeda, Janssen/Eisai, and AstraZeneca, as well as comments received from marketing authorization holders. The FDA-approved PPI labels do not mention the adverse effect at all. The FDA should consider adding SCLE as an adverse event to PPI prescription labels. This can be supplemented with a Medication Guide for patients, containing adverse event warnings and describing alternatives to PPIs.

\subsection{Clinical Practice Guidelines and Recommendations}

Risk factors for developing SCLE include being a woman of child-bearing age, periods of female hormone changes, previous drug allergies or previous SCLE episodes, sunreactive skin, exposure to ultraviolet radiation, and family history. When prescribing PPIs, especially to at-risk patients, the prescriber or the healthcare provider should ensure the patient is aware of the possibility of skin rash and SCLE and the associated symptoms. Patients should be advised to take protective measures against sun exposure, especially for bare skin. Non-allergic sunscreens or antioxidants may be applied to the skin if sun exposure cannot be avoided. If lesions occur, especially in sun-exposed areas of the skin, and if they are accompanied by arthralgia, the patient should seek medical help promptly. If the healthcare professional suspects SCLE and the patient is receiving any PPIs, the PPI should be discontinued immediately. The patient should be medically followed to observe remission of SCLE symptoms. If warranted, the patient can be treated with hydroxychloroquine, topical steroids, topical tacrolimus, and prednisolone 
[10]. For acid reflux treatment, the patient may start one of the following: alginate and bicarbonate sodium [13], magnesium carbonate, magnesium trisilicate, and magnesium hydroxide/milk of magnesia [14]. PPIs should not be restarted, even if acid reflux symptoms do not subside, as they may induce a relapse of SCLE. H2 receptor antagonists may be initiated under medical supervision and the patient followed-up for any SCLE relapse. If neither of these therapies alleviates the symptoms, nissen fundoplication is also an option to consider. Regulatory bodies such as FDA should incorporate the drug-induced adverse reaction of SCLE in prescription labels of PPIs, especially for at-risk populations.

\section{Conclusion}

Analysis of passive pharmacovigilance data confirms a statistically significant association between SCLE and PPIs. SCLE can be induced by PPIs, and patients and prescribers should be made aware of the association between the two. An increased awareness among prescribers and patients can lead to withdrawal of the offending PPI and subsequent remission of the lupus erythematosus, decreasing the morbidity of the patients.

\section{Compliance with Ethical Standards}

Ethical approval Ethical approval was not required.

Conflict of interest Nitish Aggarwal has no conflicts of interest to declare.

Funding No sources of funding were used to assist in the preparation of this study.

Open Access This article is distributed under the terms of the Creative Commons Attribution-NonCommercial 4.0 International License (http://creativecommons.org/licenses/by-nc/4.0/), which permits any noncommercial use, distribution, and reproduction in any medium, provided you give appropriate credit to the original author(s) and the source, provide a link to the Creative Commons license, and indicate if changes were made.

\section{References}

1. Cooper GS, Bynum MLK, Somers EC. Recent insights in the epidemiology of autoimmune diseases: improved prevalence estimates and understanding of clustering of diseases. J Autoimmun. 2009;33(3-4):197-207.

2. Grönhagen CM, Fored CM, Linder M, Granath F, Nyberg F. Subacute cutaneous lupus erythematosus and its association with drugs: a population-based matched case-control study of 234 patients in Sweden. Br J Dermatol. 2012;167(2):296-305.

3. Sachs G, Shin JM, Howden CW. Review article: the clinical pharmacology of proton pump inhibitors. Aliment Pharmacol Ther. 2006;23(Suppl 2):2-8.
4. US Food and Drug Administration. Questions and answers on FDA's Adverse Event Reporting System (FAERS). http://www. fda.gov/Drugs/GuidanceComplianceRegulatoryInformation/ Surveillance/AdverseDrugEffects/. Accessed 05 Mar 2016.

5. Evans SJW, Waller PC, Davis S. Use of proportional reporting ratios (PRRs) for signal generation from spontaneous adverse drug reaction reports. Pharmacoepidemiol Drug Saf. 2001;10:483-6.

6. US National Library of Medicine, National Institutes of Health. PubMed [database]. http://www.ncbi.nlm.nih.gov/ pubmed.Accessed 5 Jan 2016.

7. Sandholdt LH, Laurinaviciene R, Bygum A. Proton pump inhibitor-induced subacute cutaneous lupus erythematosus. $\mathrm{Br} \mathrm{J}$ Dermatol. 2014;170(2):342-51.

8. Dam C, Bygum A. Subacute cutaneous lupus erythematosus induced or exacerbated by proton pump inhibitors. Acta Derm Venereol. 2008;88(1):87-9.

9. Brinker AD, Lyndly J, Tonning J, Moeny D, Levine JG, Avigan MI. Profiling cumulative proportional reporting ratios of druginduced liver injury in the FDA Adverse Event Reporting System (FAERS) database. Drug Saf. 2013;36(12):1169-78.

10. Lowe G, Henderson CL, Grau RH, Hansen CB, Sontheimer RD. A systematic review of drug-induced subacute cutaneous lupus erythematosus. Br J Dermatol. 2011;14(3):465-72.

11. European Medicines Agency. PRAC recommendations on signals: adopted at the PRAC meeting of 6-9 July 2015. http://www. ema.europa.eu/docs/en_GB/document_library/PRAC_ recommendation_on_signal/2015/07/WC500190054.pdf. Accessed 5 Jan 2016.

12. Medicines and Healthcare products Regulatory Agency. Drug safety update. Proton pump inhibitors: very low risk of subacute cutaneous lupus erythematosus (8 September 2015). https://www. gov.uk/drug-safety-update/proton-pump-inhibitors-very-lowrisk-of-subacute-cutaneous-lupus-erythematosus. Accessed 5 Jan 2016.

13. De Ruigh A, Roman S, Chen J, Pandolfino JE, Kahrilas PJ. Gaviscon double action liquid (antacid and alginate) is more effective than antacid in controlling post-prandial oesophageal acid exposure in GERD patients: a double-blind crossover study. Aliment Pharmacol Ther. 2014;40(5):531-7.

14. CFR331, Electronic Code of Federal Regulations, Title 21, Chapter 1, Subchapter D, Subpart B, 331 US Government Publishing Office. http://www.ecfr.gov/cgi-bin/text-idx?SID= $7921869728 \mathrm{~d} 8 \mathrm{c} 21 \mathrm{c} 6846 \mathrm{aafab} 37668 \mathrm{c} 9 \& \mathrm{mc}=$ true $\&$ node $=\mathrm{pt} 21.5$. 331\&rgn=div5. Accessed 12 Mar 2016.

15. Reich A, Maj J. Subacute cutaneous lupus erythematosus due to proton pump inhibitor intake: case report and literature review. Arch Med Sci. 2012;8(4):743-7.

16. Toms-Whittle LM, John LH, Buckley DA. Drug-induced subacute cutaneous lupus erythematosus associated with omeprazole. Clin Exp Dermatol. 2011;36(3):281-3.

17. Correia O, Lomba Viana H, Azevedo R, Delgado L, Polónia J. Possible phototoxicity with subsequent progression to discoid lupus following pantoprazole administration. Clin Exp Dermatol. 2001;26(5):455-6.

18. Bracke A, Nijsten T, Vandermaesen J, Meuleman L, Lambert J. Lansoprazole-induced subacute cutaneous lupus erythematosus: two cases. Acta Derm Venereol. 2005;85(4):353-4.

19. Jones EK, Mingioni N, Lee JB. Widespread scaly eruption in a patient with multiple comorbidities. JAMA. 2015;314(16):1740-1.

20. Hung R, Sangle SR, Benton E, D’Cruz DP, McGibbon D. Proton pump inhibitor-induced subcutaneous lupus erythematosus in a patient with systemic lupus erythematosus. Clin Exp Dermatol. 2015;40(7):808-9.

21. Almebayadh M, Regnier-Rosencher E, Carlotti A, Goulvestre C, Le Guern V, Mouthon L, Avril MF, Dupin N. Subacute cutaneous 
lupus erythematosus induced and exacerbated by proton pump inhibitors. Dermatology. 2013;226(2):119-23.

22. Wee JS, Natkunarajah J, Marsden RA. A difficult diagnosis: drug-induced subacute cutaneous lupus erythematosus (SCLE) triggered by omeprazole in a patient with pre-existing idiopathic SCLE. Clin Exp Dermatol. 2012;37(4):445-6.

23. Alcántara-González J, Truchuelo-Díez MT, González-García C, Jaén Olasolo P. Esomeprazole-induced subacute cutaneous lupus erythematosus. Actas Dermosifiliogr. 2011;102(8):638-40.
24. Mankia SK, Rytina E, Burrows NP. Omeprazole-induced subacute cutaneous lupus erythematosus. Clin Exp Dermatol. 2010;35(3):e1-2.

25. Drago F, Javor S, Ciccarese G, Cozzani E, Parodi A. Subacute cutaneous lupus erythematosus induced by lansoprazole. Eur J Clin Pharmacol. 2015;71(6):767-8.

26. Panting KJ, Pinto M, Ellison J. Lansoprazole-induced subacute cutaneous lupus erythematosus. Clin Exp Dermatol. 2009;34(6):733-4. 